\title{
A prospective large-scale study of methods for the detection of latent Mycobacterium tuberculosis infection in refugee children
}

\author{
Michaela Lucas, ${ }^{1}$ Pam Nicol, ${ }^{2}$ Elizabeth McKinnon, ${ }^{3}$ Rebecca Whidborne, ${ }^{1}$ \\ Andrew Lucas, ${ }^{3}$ Aesen Thambiran, ${ }^{4}$ David Burgner, ${ }^{5}$ Justin Waring, ${ }^{6}$ Martyn French ${ }^{7}$
}

'Department of Clinical Immunology, Royal Perth Hospital and Pathwest Laboratory Medicine, Perth, Western Australia ${ }^{2}$ School of Paediatrics and Child Health, University of Western Australia, Perth, Western Australia

${ }^{3}$ Centre for Clinical Immunology and Biomedical Statistics, Murdoch University, Perth, Western Australia

${ }^{4}$ Migrant Health Unit, North Metropolitan Area Health

Service, Perth, Western Australia

${ }^{5}$ Murdoch Children's Research Institute, Royal Children's Hospital, Parkville, Victoria ${ }^{6}$ TB Control Program, North Metropolitan Area Health Service, Perth, Western Australia

${ }^{7}$ School of Pathology and Laboratory Medicine, University of Western Australia, Perth, Western Australia

\section{Correspondence to}

Dr Michaela Lucas, Department of Clinical Immunology, Royal Perth Hospital, GPO Box X2213, Perth WA 6847, Australia; michaela.lucas@health.wa.gov. au

Received 24 September 2009 Accepted 18 February 2010

\begin{abstract}
Background Diagnosis of latent tuberculosis infection (LTBI) is a cornerstone of the health assessment of resettled high incidence populations, particularly in children. Two blood-based interferon $\gamma$ release assays (IGRAs), T-SPOT.TB and QFT-Gold in-tube (QFT-GIT), have greater sensitivity and specificity than the tuberculin skin test (TST), but their performance as screening tools for LTBI in children, especially refugee children, remains unclear.
\end{abstract}

Methods 524 African and ethnic Burmese children, including 107 under 3 years of age, were prospectively enrolled in a comparison of the T-SPOT.TB and OFT-GIT. The TST was also performed in 342 of the children.

Results The T-SPOT.TB and QFT-GIT had similar rates of positivity ( $8 \%$ and $10 \%$, respectively) and showed good concordance when both tests gave definitive results $(\kappa=0.78 ; p<0.0001)$. However, the IGRAs had significant failure rates: $15 \%$ of QFT-GIT gave indeterminate results due to failed mitogen response and $14 \%$ of T-SPOT.TB results were inconclusive, largely because of insufficient mononuclear leucocyte yields. Failure of the QFT-GIT mitogen response was associated with African ethnicity and co-morbid infections, particularly with helminths. The TST results showed poor concordance $(\sim 50 \%)$ with both IGRAs.

Conclusions It is reasonable to screen using either IGRA with follow-up by the alternative if the test fails. In general, the OFT-GIT is the preferred option for nonAfrican populations but the T-SPOT.TB is recommended when there are epidemiological and/or clinical high risk factors for TB infection. However, both IGRAs have methodological and performance characteristics that limit their usefulness in refugee children, highlighting the need for continued development of screening strategies.

\section{INTRODUCTION}

Increasing migration from resource-poor to resource-rich countries has important public health implications for countries of resettlement. Diagnosis and treatment of Mycobacterium tuberculosis infection is arguably the most important health intervention for migrants from high incidence settings, to low incidence countries. Australia is one such low incidence country, in which $85 \%$ of tuberculosis (TB) notifications in 2006 were for people born overseas. ${ }^{1}$ Predeparture chest radiography is used to screen for active pulmonary $\mathrm{TB}$ but cannot identify latent TB infection (LTBI). The overall lifetime risk of LTBI reactivation is $\sim 10 \%$, but is increased $4-5$ times in infants and young children, ${ }^{2}$ who are also at greater risk of extrapulmonary $\mathrm{TB}$, including $\mathrm{TB}$ meningitis. ${ }^{3}$ Diagnosing LTBI in migrants from high incidence countries is a cornerstone of the health assessment following resettlement in low incidence countries. The optimal method for the diagnosis of LTBI in children, particularly resettled refugee children, is unknown.

The tuberculin skin test (TST) is an established method for detecting immunological memory to mycobacterial antigens but has well-recognised limitations: poor specificity due to cross-reactivity with both non-tuberculous mycobacteria (NTM) and BCG, and a requirement for two patient visits. ${ }^{4}$ Blood-based interferon $\gamma$ (IFN $\gamma$ ) release assays (IGRAs) have shown high specificity for $M$ tuberculosis infection in adults, especially in BCG-vaccinated populations. ${ }^{5}$ Two IGRAs are currently available for diagnostic use, the Quantiferon-TB gold in-tube (OFT-GIT; Cellestis, Carnegie, Australia) and the T-SPOT.TB (Oxford Immunotech, Oxford, UK). Both measure T cell IFNy production in response to antigens that are not present in BCG and are predominantly $M$ tuberculosis specific. T-SPOT.TB is an enzyme-linked immunospot (ELISpot) assay that measures the response to two antigens-early-secreted antigenic target 6 (ESAT-6) and culture filtrate protein 10 (CFP-10). The OFT-GIT assay is a whole blood IGRA assay that includes an additional $M$ tuberculosis-specific antigen (TB7.7).

In the few head-to head comparisons of the T-SPOT.TB and OFT-GIT assays and TST there has been limited focus on high incidence paediatric populations. ${ }^{6-8}$ We conducted a prospective comparative study of IGRAs and TST for the diagnosis of LTBI in 524 recently resettled refugee children, including 182 children $<5$ years of age, from countries with a high prevalence of $M$ tuberculosis. Our findings demonstrate the influence of age, ethnicity and clinical status on IGRA results and the limitations of using these tests in refugee children.

\section{METHODS \\ Study population}

Children aged from 5 months to 16 years were consecutively and prospectively enrolled between January 2007 and March 2008 from refugee families attending the Migrant Health Unit (MHU), Perth, Western Australia. In 2007, there were 1550 humanitarian entrants settling in Western Australia 
of whom 1237 (80\%) were assessed in the MHU. The remaining $20 \%$ were mainly sponsored refugees whose sponsors may contact the MHU. Consent was obtained from 524 parents or adult guardians via an interpreter and assent was obtained from older adolescents. Consent was obtained from all but six adolescents (99\%). One HIV-positive child was excluded. Approval was obtained from the Royal Perth Hospital Ethics Committee in consultation with the local tertiary paediatric hospital. Positive or indeterminate IGRA results were reported to the MHU doctor to aid clinical management.

Clinical data collected included date of birth, sex, height, weight, country of birth, country of transit, history of or contact with $\mathrm{TB}$, co-morbidities, regular medication and recent treatment for helminth infection. Data collected as part of the initial health assessment included chest X-ray (children $\geq 11$ years old and a positive TB screening test or clinical indications), schistosomiasis, strongyloides, hepatitis B (HBV), hepatitis C (HCV) and HIV serology, full blood count, serum albumin, serum ferritin, malaria status, thyroid-stimulating hormone (TSH), vitamin D, calcium and phosphorus. Data were ascertained on those children for whom venepuncture failed.

\section{T-SPOT.TB assay}

In keeping with the manufacturer's instructions, $4 \mathrm{ml}$ of blood were drawn for the T-SPOT.TB assay, except for children $<2$ years when $2-3 \mathrm{ml}$ were drawn depending on ease of venepuncture. Inconclusive assays were defined by an inability to complete the test due to inadequate peripheral blood mononucear cell (PBMC) yield after PBMC separation, high background, machine failure or red blood cell contamination. Indeterminate assays were defined as a low mitogen-positive control response or a high response to the negative control.

\section{OFT-GIT assay}

A $3 \mathrm{ml}$ aliquot of blood was drawn from all study children and the assay was performed according to the manufacturers' protocols; indeterminate assays were defined as a high IFN $\gamma$ response to the negative control or a low IFN $\gamma$ response to

Table 1 Cohort demographics and clinical summaries

\begin{tabular}{|c|c|c|c|}
\hline total $\mathbf{N}=524$ & $\begin{array}{l}\text { Africa } \\
\mathrm{n}=411^{*}\end{array}$ & $\begin{array}{l}\text { Asia } \\
n=113 \dagger\end{array}$ & p Value $\neq$ \\
\hline \multicolumn{4}{|l|}{ Demographics } \\
\hline Age (median (IQR) years) & $7.1(3.6-11.0)$ & $7.8(2.8-11.9)$ & NS \\
\hline Body mass index (median (IOR)) & $16.5(15.3-18.1)$ & $16.2(14.9-18.7)$ & NS \\
\hline Male $(\%$ of $n)$ & $47 \%$ & $51 \%$ & NS \\
\hline \multicolumn{4}{|l|}{ Clinical history (\% of $n)$} \\
\hline Cough & $1.9 \%$ & $0 \%$ & NS \\
\hline Underweight§ & $8 \%$ & $12 \%$ & NS \\
\hline \multicolumn{4}{|l|}{ Household TB contact: } \\
\hline Definite or suspected & $6 \%$ & $17 \%$ & 0.0001 \\
\hline (definite; suspected) & $(3 \% ; 3 \%)$ & $(11 \% ; 6 \%)$ & \\
\hline \multicolumn{4}{|l|}{ Clinical investigations $(n ; \%$ of $n$ ) } \\
\hline Chest radiograph & $\mathrm{n}=101$ & $\mathrm{n}=34$ & \\
\hline Abnormal & $5 \%$ & $3 \%$ & NS \\
\hline Malaria & $\mathrm{n}=378$ & $\mathrm{n}=108$ & \\
\hline Positive thick/thin films & $4 \%$ & $1 \%$ & NS \\
\hline Hepatitis B & $\mathrm{n}=288$ & $\mathrm{n}=68$ & \\
\hline Surface antigen positive & $2 \%$ & $3 \%$ & NS \\
\hline Hepatitis C & $\mathrm{n}=384$ & $\mathrm{n}=108$ & \\
\hline Serum antibody positive & $2 \%$ & $1 \%$ & NS \\
\hline Schistosomiasis & $\mathrm{n}=389$ & $\mathrm{n}=42$ & \\
\hline $\begin{array}{l}\text { Positive (indirect haemagglutination } \\
\text { titre }>16 \text { ) }\end{array}$ & $18 \%$ & $0 \%$ & 0.006 \\
\hline Strongyloides & $\mathrm{n}=387$ & $\mathrm{n}=108$ & \\
\hline $\begin{array}{l}\text { Positive (>0.45, ElA expressed as } \\
\text { absorbance) }\end{array}$ & $2 \%$ & $0 \%$ & NS \\
\hline BCG & $\mathrm{n}=411$ & $\mathrm{n}=112$ & \\
\hline Scar/history & $67 \%$ & $77 \%$ & NS \\
\hline \multicolumn{4}{|l|}{ Laboratory investigations (median (IOR)) } \\
\hline Lymphocytes $\left(\times 10^{9} /\right)$ & $3.0(2.3-4.1)$ & $3.0(2.4-4.0)$ & NS \\
\hline Neutrophils $\left(\times 10^{9} / \mathrm{l}\right)$ & $2.1(1.7-2.8)$ & $3.7(3.1-4.5)$ & $<0.0001$ \\
\hline Eosinophils $\left(\times 10^{9} / \mathrm{I}\right)$ & $0.2(0.1-0.3)$ & $0.3(0.2-0.5)$ & $<0.0001$ \\
\hline Haemoglobin $(g / l)$ & $121(111-128)$ & $123(117-130)$ & 0.02 \\
\hline Albumin $(\mathrm{g} / \mathrm{l})$ & $45(43-47)$ & $47(45-48)$ & $<0.0001$ \\
\hline Vitamin $D(\mu \mathrm{mol} / \mathrm{l})$ & $57(43-70)$ & $46(32-60)$ & $<0.0001$ \\
\hline
\end{tabular}

${ }^{*}$ Country of birth: Sudan $(24 \%)$, Uganda (12\%) Tanzania (11\%), DRC (10\%), Kenya (5\%), Ethiopia (5\%), other (33\%). Country of transit: Uganda (21\%), Tanzania (12\%), Sudan (10\%), Guinea (9\%), Kenya (8\%) Egypt $(7 \%)$, other $(33 \%)$.

† Country of birth: Thailand (52\%), Myanmar (36\%), Malaysia (7\%), India (4\%), Indonesia (2\%). Country of transit: Thailand (64\%), Malaysia (25\%), India (6\%), Myanmar (4\%), Indonesia (2\%).

‡Comparison of African vs Asian: $\chi^{2}$ test used for dichotomous variables; $t$ test used for continuous variables, with log transformation of laboratory marker values. NS, p value $>0.1 ; \mathrm{NS} \uparrow, 0.05<\mathrm{p}$ value $<0.1$

$\S<5$ th percentile of body mass index for age charts compiled by the World Health Organization $(0-5$ years) or the National Center for Health Statistics ( $>5$ years)

EIA, enzyme immunoassay; TB, tuberculosis. 
Table 2 Summary of test results according to region of origin

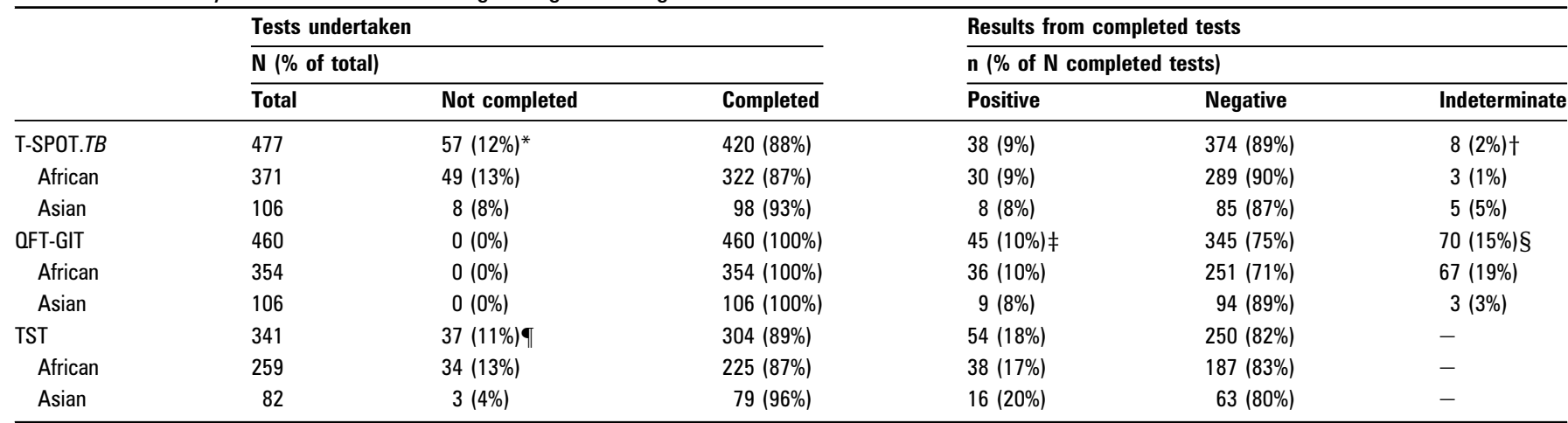

*46 $(9.6 \%)$ insufficient peripheral blood mononuclear cells (PBMCs), 5 (1\%) high background, $3(0.6 \%)$ haemolysed, 3 (0.6\%) other technical problems.

†All failed negative control.

$\neq 6(1 \%)$ low positive control, but QFT-GIT positive according to manufacturer's directions.

$\S 66(14.3 \%)$ low positive control, $4(0.7 \%)$ failed negative control.

TFollow-up reading not obtained.

mitogen stimulation in the absence of a positive antigen response.

\section{Tuberculin skin test}

TST was performed with purified protein derivative (PPD) by administration of 5 tuberculin units following the Mantoux method. ${ }^{4}$ The transverse diameter of skin induration was measured at $48-72 \mathrm{~h}$. The presence of a BCG vaccination scar and history of BCG vaccination was recorded and results interpreted using Australian guidelines. ${ }^{69}$ Generally, a $10 \mathrm{~mm}$ cut-off was used for positivity given that all children originated from high prevalence countries. A $15 \mathrm{~mm}$ cut-off was used if children were $<5$ years old and had received BCG. Five $\mathrm{mm}$ was subtracted from these cut-off values for children at increased risk for $M$ tuberculosis infection (such as household contacts) and for those $>1$ year of age, giving a cut-off value of 5 or $10 \mathrm{~mm}$, respectively for these groups.

\section{Statistical analysis}

Demographic variables and laboratory markers were compared across independent sample subgroups by means of $\chi^{2}$ tests or two-sample $t$ tests, with log transformation of skewed data where necessary. Logistic regression was used to assess predictors of dichotomous IGRA and TST outcomes. Cohen's $\kappa$ and proportions of specific agreement were used as measures of concordance between test results, ${ }^{10}{ }^{11}$ and the McNemar test was used to assess the superiority of one assay over the other. Mitogen control responses were analysed by censored-normal regression methods. Analyses were carried out using S-PLUS 8.0.4 for Windows (Insightful, Seattle, Washington, USA).

\section{FINDINGS}

Demographic and clinical data are summarised in table 1 . There were similar numbers of boys and girls (48\% male), and the majority were of African origin (African 78\%, Asian 22\%). A significantly higher proportion of Asian children had a known or suspected household TB contact compared with African children (19/113 vs 22/411, $\mathrm{p}=0.0001)$.

A total of 477 T-SPOT.TB and 460 OFT-GIT were attempted. Insufficient blood volume, particularly from younger children, was the main reason that both IGRA tests were not attempted in all children. The TST was attempted in 341 children; in 37 $(11 \%)$ reading was not possible due to absence of the child at the scheduled clinic or home follow-up.

Results were positive for $9 \%$ of the completed T-SPOT.TB assays, $10 \%$ of the OFT-GIT assays and $18 \%$ of TSTs (table 2).
Figure 1 Histograms showing the distributions of IGRA (interferon $\gamma$ release assays) results for attempted tests according to region of origin and serological evidence of helminth infection. Proportions of positive results were comparable across region of origin for both T-SPOT.TB $(\mathrm{p}>0.9)$ and QFT-GIT $(p=0.8)$. QFT-GIT indeterminacy was more prevalent in African samples $(p=0.0001)$, especially amongst children with helminth infection $(\mathrm{p}<0.0001)$.
Region of Origin

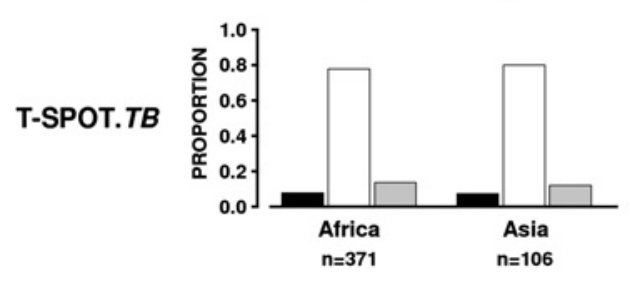

Region of Origin

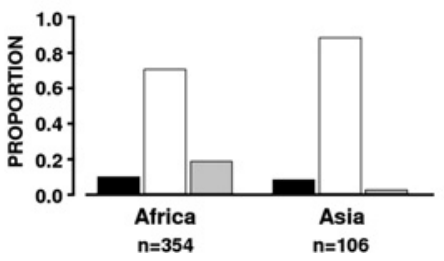

Schistosomiasis/Strongyloides in Africans

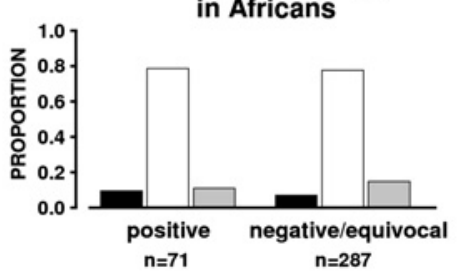

Positive

Negative

Indeterminate not completed

Schistosomiasis/Strongyloides
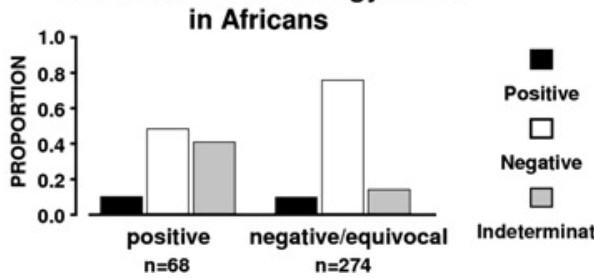
Table 3 Demographic variables as predictors of a positive test result

\begin{tabular}{|c|c|c|c|c|c|c|}
\hline \multirow[b]{2}{*}{ Variable } & \multicolumn{2}{|l|}{ T-SPOT.TB } & \multicolumn{2}{|l|}{ OFT-GIT } & \multicolumn{2}{|l|}{ TST } \\
\hline & OR (95\% Cl) & p Value & OR (95\% Cl) & p Value & OR $(95 \% \mathrm{Cl})$ & p Value \\
\hline Age: $5-7$ years vs $<5$ years & $1.9(0.4$ to 9.0$)$ & NS & $1.6(0.5$ to 5.6$)$ & NS & $1.1(0.4$ to 3.0$)$ & NS \\
\hline $12-16$ years vs $<5$ years & $10.2(2.9$ to 36.8$)$ & $<0.001$ & $5.7(2.0$ to 16.2$)$ & 0.001 & $3.0(1.3$ to 6.7$)$ & 0.008 \\
\hline $\begin{array}{l}\text { Household TB contact: definite/suspected } \\
\text { vs none }\end{array}$ & $2.5(0.9$ to 6.5$)$ & $\mathrm{NS}^{*}$ & $2.4(1.0$ to 5.8$)$ & $\mathrm{NS}^{*}$ & $4(1.7$ to 9.5$)$ & 0.001 \\
\hline BCG vaccination: scar/history vs none & $1.8(0.8,4.0)$ & NS & $1.7(0.8,3.6)$ & NS & $1.7(0.8$ to 3.5$)$ & $\mathrm{NS}^{*}$ \\
\hline Region of origin: African vs Asian & $1.1(0.5$ to 2.5$)$ & NS & $1.2(0.6$ to 2.7$)$ & NS & $0.8(0.4$ to 1.6$)$ & NS \\
\hline
\end{tabular}

univariate logistic regression used for all analyses. NS $p$ value $>0.05 ; N^{*} 0.05<p$ value $\leq 0.1$

TB, tuberculosis; TST, tuberculin skin test.

For all three tests, the proportions of positive results were similar for children of African and Asian origin (figure 1).

The youngest age for which a positive result was obtained was 37, 34 and 7 months for the T-SPOT.TB, OFT-GIT and TST, respectively. For all tests, increasing positivity was associated with increasing age (table 3). Correspondingly, the mean age of children with positive tests was higher than that of children with negative tests (mean (SD): T-SPOT.TB 11.2 (3.7) vs 7.7 (4.2), $\mathrm{p}<0.0001$; OFT-GIT 10.5 (4.0)) vs 7.8 (4.3), $\mathrm{p}=0.0001$; TST 9.1 (5.1) vs 7.3 (4.3), $\mathrm{p}=0.02$ ). Another significant predictor of a positive test result was definite or suspected household TB contact, but not previous BCG vaccination, presence of cough or region of origin (table 3 ).

Neither IGRA was significantly superior in terms of overall positivity (table 4), considered as the proportion of either completed tests or all tests attempted. There was good concordance when both tests gave definitive results $(\kappa=0.78$ (0.67-0.89); $81 \%$ positive and $97 \%$ negative agreement). OFTGIT had significantly more indeterminate results than completed T-SPOT.TB $(\mathrm{p}<0.0001)$, although the overall proportions of inconclusive results, taking into account all attempted, but failed T-SPOT.TB tests, were similar (table 4).

Of T-SPOT.TB tests that failed to yield a definitive result, relatively few were truly indeterminate (8/65, table 2$)$. The majority of inconclusive T-SPOT.TB assays were attributable to inadequate PBMC yield. Other technical problems (high background, machine failure and red blood cell contamination) contributed to a lesser extent. Compared with children with negative T-SPOT.TB results, those with insufficient PBMCs tended to be younger (mean (SD): 6.1 (3.9) vs 7.7 (4.2), $p=0.01$ ) but did not significantly differ in laboratory parameters,

Table 4 A comparison of the T-SPOT.TB and QFT-GIT assays where both tests were undertaken

\begin{tabular}{lllll}
\hline & OFT-GIT & & & \\
\cline { 2 - 4 } & Positive & Negative & Indeterminate & Total \\
\hline T-SPOT.TB positive & 31 & 6 & 0 & $37(8.1 \%)^{*}$ \\
T-SPOT.TB negative & 9 & 289 & 62 & $360(78 \%)$ \\
T-SPOT.TB inconclusive & $3+2$ & $43+6$ & $8+0$ & $62(14 \%) \neq$ \\
Total & 45 & 344 & 70 & $\mathrm{n}=459$ \\
& $(9.8 \%)^{*}$ & $(75 \%)$ & $(15 \%) \dagger$ & \\
\hline
\end{tabular}

Comparisons are based on the McNemar test for paired proportions.

A T-SPOT.TB result was inconclusive if the test was not completed or the result was indeterminate.

*Proportions positive: $9 \%$ (of completed T-SPOT.TB) and $8 \%$ (of attempted T-SPOT.TB) vs $10 \%$ (QFT-GIT), $p=0.3$ and $p=0.1$, respectively.

tx+y: T-SPOT.TB number not completed + number indeterminate

$\ddagger$ Proportions indeterminate: $2 \%$ (T-SPOT.TB) vs $15 \%$ (QFT-GIT), $\mathrm{p}<0.0001$. Proportions

inconclusive (not completed or indeterminate): $14 \%$ (T-SPOT.TB) vs $15 \%$ (QFT-GIT), $p=0.5$. including lymphocytes, white cells or neutrophil counts. With model adjustment for age, however, lymphocyte and white cell counts were lower $(p=0.0001$ and $p=0.01)$ and ferritin levels higher $(p=0.03)$ in children with an insufficient PBMC yield. Region of origin and indicators of other infections were not predictive of insufficient PBMCs or an indeterminate T-SPOT.TB result.

In contrast to the findings for the T-SPOT.TB assay, all inconclusive OFT-GIT results were truly indeterminate, with most resulting from a low mitogen-positive control response. Mitogen responses were significantly lower for African children $(p<0.0001)$. Correspondingly, indeterminate OFT-GIT assay results were much more prevalent amongst African compared with Asian children $(p=0.0001$, table 2, figure 1$)$. African children with serological evidence of either schistosomiasis or strongyloides infection had considerably increased odds of an indeterminate OFT-GIT result (OR 4.7, $p<0.0001$, vs no infection) (figure 1b) and indeterminancy was associated with higher eosinophil count $(p=0.003)$. Amongst those with schistosomiasis and/or strongyloides infection, children with indeterminate QFT-GIT results were less likely to have received predeparture treatment for helminth infection (16/48 vs 31/33, $\mathrm{p}=0.003$ ). Of African children not positive for helminth infection, those with indeterminate results were younger than those with negative results $(\mathrm{p}=0.01)$ and the odds for indeterminacy were higher for those testing positive for malaria or either HBV or HCV (OR 3.4, $p=0.02$ ). Vitamin D levels in the African children were not correlated with magnitude of responses to mitogen $(p=0.3)$. No Asian children had a diagnosed helminth infection, and the three Asian children with an indeterminate OFT-GIT result did not have malaria or hepatitis virus infection.

The most striking difference between the IGRAs was the likelihood of returning a final conclusive result once the test was attempted, rather than overall positivity. We therefore considered T-SPOT.TB vs OFT-GIT in light of the strong demographic predictors for OFT-GIT indeterminacy. In particular, we analysed relative proportions of both positive and inconclusive results within three demographic groups defined by: (1) African children without diagnosed chronic infections: malaria, hepatitis B or C, schistosomiasis and/or strongyloides infection; (2) African children with at least one of these infections; and (3) Asian children (table 5)

In terms of the likelihood of the IGRAs giving a definitive result, the OFT-GIT was the superior assay for the Asian children, whereas the T-SPOT.TB performed better in African children with other infections. For African children without identified co-morbid infections, neither assay was clearly superior in its likelihood to provide a definitive result. 
Table 5 Comparisons of results for the T-SPOT.TB and QFT-GIT assays, where both tests were undertaken, according to region of origin and presence of diagnosed co-morbidities

\begin{tabular}{lcll}
\hline & QFT-GIT & QFT-GIT & QFT-GIT \\
\cline { 2 - 4 } & Positive & Negative & Indeterminate \\
\hline African without co-morbities* & $(\mathrm{n}=263)$ & & \\
T-SPOT.TB positive & $15(6 \%)$ & $3(1 \%)$ & $0(0 \%)$ \\
T-SPOT.TB negative & $6(2 \%)$ & $171(65 \%)$ & $28(11 \%)$ \\
T-SPOT.TB inconclusive & $3(1 \%)$ & $32(12 \%)$ & $5(2 \%)$ \\
African with co-morbities $\dagger$ & $(\mathrm{n}=90)$ & & \\
T-SPOT.TB positive & $10(11 \%)$ & $1(1 \%)$ & $0(0 \%)$ \\
T-SPOT.TB negative & $2(2 \%)$ & $37(41 \%)$ & $31(34 \%)$ \\
T-SPOT.TB inconclusive & $0(0 \%)$ & $6(7 \%)$ & $3(3 \%)$ \\
Asian $\neq(\mathrm{n}=106)$ & & & \\
T-SPOT.TB positive & $6(6 \%)$ & $2(2 \%)$ & $0(0 \%)$ \\
T-SPOT.TB negative & $1(1 \%)$ & $81(76 \%)$ & $3(3 \%)$ \\
T-SPOT.TB inconclusive & $2(2 \%)$ & $11(10 \%)$ & $0(0 \%)$ \\
\hline
\end{tabular}

Diagnosed co-morbidities included malaria, hepatitis or helminth infection (schistosomiasis/ strongyloides).

An inconclusive T-SPOT.TB result was one that was either indeterminate or where the test was not completed.

Comparisons are based on the McNemar test for paired proportions of T-SPOT.TB vs QFTGIT:

*Proportions positive: $7 \%$ vs $9 \%, p=0.1$. Proportions not completed or indeterminate: $15 \%$ vs $13 \%, p=0.4$.

†Proportions positive: $12 \%$ vs $13 \%, p>0.9$. Proportions not completed or indeterminate: $10 \%$ vs $38 \%, p=0.0001$.

†Proportions positive: $8 \%$ vs $8 \%, p>0.9$. Proportions not completed or indeterminate: $12 \%$ vs $3 \%, p=0.02$.

There was only moderate concordance between the TST and either IGRA (T-SPOT.TB: $\kappa=0.45(0.38-0.53)$; OFT-GIT: $\kappa=0.46$ (0.39-0.53); figure 2). More particularly, amongst children having valid results for all three tests $(n=239), 24 / 47(51 \%)$ of children with a positive TST result were negative for both IGRAs and, conversely, 9/30 (30\%) of children with a positive IGRA had a negative TST. Figure 3 highlights the discordant positivity between TST and IGRA results. Notably, five of nine children with at least one positive IGRA result but a negative TST had no induration measurable. Positive agreement between the TST and both IGRAs was higher for African children

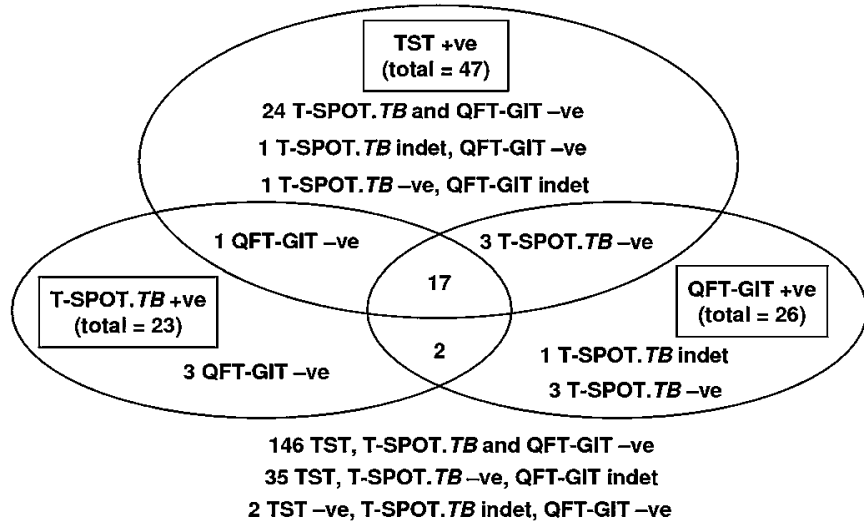

Figure 2 Venn diagram showing the distribution of results (positive, negative or indeterminate), where available, for all three tests. TST, tuberculin skin test.

(T-SPOT.TB, 57\%; OFT-GIT, 56\%) than for Asian children (T-SPOT.TB, 40\%; OFT-GIT, 45\%).

\section{DISCUSSION}

This prospective study is the first large-scale investigation of the clinical utility of currently available immunological tests for LTBI in resettled refugee children. There is a lack of evidence regarding the optimal diagnostic test for LTBI from sufficiently powered cohort studies of children, particularly those $<5$ years old. These data are essential to balance the need for treatment of LTBI against unnecessary interventions and overtreatment in this vulnerable population. Our primary aim was therefore to investigate whether the superior performance attributes of the T-SPOT.TB assay over the OFT-GIT assay, reported in other settings, were also found in refugee children.

The two IGRAs showed similar positivity rates across all age groups. This suggests that in contrast to findings from immunosuppressed adults, ${ }^{12}$ the greater intrinsic sensitivity of the $\mathrm{T}$ SPOT.TB for antigen-specific $T$ cells may be less critical for the
Figure 3 Tuberculin skin test (TST) induration plotted against age according to history of BCG and household tuberculosis (TB) contact (definite or suspected). Age-appropriate cut-offs for definition of TST positivity are indicated by horizontal lines and differentiation of IGRA (interferon $\gamma$ release assays) positivity by a symbol. Discordancy between TST and IGRA results is notable, with only $54 \%$ positive agreement observed.
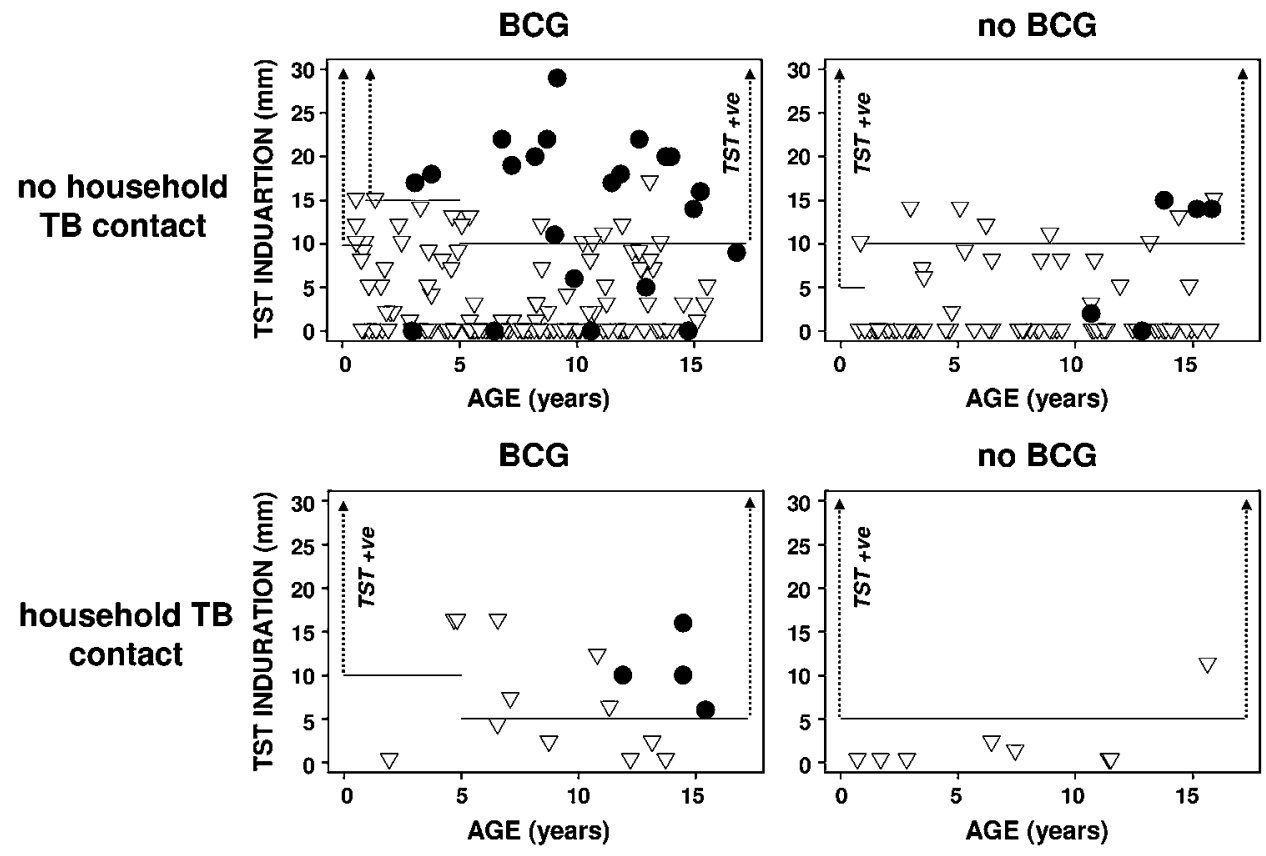

$\nabla$ neither IGRA positive 
detection of $M$ tuberculosis-specific responses in children of any age. Furthermore, the inclusion of an additional antigen in the OFT-GIT may contribute to an increase in positivity. Despite reasonable concordance of positive results between the IGRAs, there were a number of discordant positive results. Therefore, testing with the alternative IGRA may be indicated where clinical suspicion of $M$ tuberculosis infection is high. The decreased positivity associated with younger age observed for both of these assays and the TST indicates that the sensitivity of all tests may be reduced in younger children, or may simply reflect less time for potential exposure to $M$ tuberculosis infection. Our data and published data are insufficient to make definitive recommendations about age-related limitations of IGRAs. However, it should be noted that IGRAs are able to demonstrate $\mathrm{TB}$ in children $<12$ months old. ${ }^{13} 14$

Both IGRAs gave an unacceptably high proportion of inconclusive results. Failed tests were the primary cause of inconclusive T-SPOT.TB assays whereas indeterminate results were the primary cause of inconclusive OFT-GIT assays. However, an inconclusive test result with one IGRA was usually associated with a valid result for the other.

An inadequate $\mathrm{T}$ cell response to the mitogen was the predominant reason for OFT-GIT indeterminacy. However, 6/72 children with a low mitogen response were considered OFT-GIT positive based on their positive response to $M$ tuberculosis antigens. Low mitogen responses were observed predominantly in African children, and were particularly associated with serological evidence of a helminth infection and, to a lesser extent, with malaria, HBV or HCV infection. Reduced mitogen responsiveness of T cells from African children in a whole blood IGRA might have several causes. Infection with parasites, especially helminths, might polarise circulating $\mathrm{T}$ cells towards $\mathrm{T}$ helper 2 (Th2) responses, resulting in impaired Th1 responses, increased regulatory $\mathrm{T}$ cell activity and lower IFN $\gamma$ responses. ${ }^{15}$ Parasitic infections may cause immune activation and associated production of immunoglobulin $\mathrm{E}$ ( $\mathrm{IgE}$ ) antibodies, ${ }^{16}$ which can result in monocyte production of immunosuppressant factors, such as prostaglandin E2, which inhibit $\mathrm{T}$ cell proliferation. ${ }^{17}{ }^{18}$ Finally, it might reflect early life environmental exposure to pathogens. This is in keeping with the differences in T cell memory subsets, especially higher numbers of $\mathrm{T}$ cells with an effector memory immunophenotype and lower numbers of $T$ cells with a central memory immunophenotype, as described in Malawian as compared with UK adolescents, despite no differences in lymphocyte populations in cord blood from these populations. ${ }^{19}$ It has been suggested that these differences result from greater exposure to early-life infections in the African environment. ${ }^{20}$ However, IFN $\gamma$ responses to the mitogen in African children were not reduced in the T-SPOT.TB assay and it is likely that the natural exposure to infective pathogens in Asian children is also high. Given previous data suggesting that vitamin D levels affect mitogen responses, ${ }^{21}$ we examined the relationship between vitamin $\mathrm{D}$ levels and the magnitude of the mitogen response amongst the African children, but could not detect a correlation. The issue of low mitogen responses resulting in indeterminate QFT-GIT results in African children or those with active infection might be overcome by redefining the cut-offs of the positive mitogen control in this population.

The most common cause of an inconclusive outcome for the T-SPOT.TB assay was failure to isolate sufficient PBMCs. A high rate of uninterpretable results in very young children has been previously described for the T-SPOT.TB assay, although the number of children $<3$ years old in the previous study was small. ${ }^{6}$ Technical difficulties in obtaining sufficient PBMCs to complete the T-SPOT.TB assay remain a challenge, especially in samples from young children. PBMC separation protocols optimised for the larger blood volumes and lower cell counts of adults may not be suitable for children. The sample preparation protocol might therefore be modifiable in children and may decrease these assay failures. Importantly, the T-SPOT.TB assay had far fewer true indeterminate results than the QFT-GIT assay (table 2), which may be due to the greater intrinsic sensitivity of the ELISpot assay per se or due to an unknown advantage of using purified PBMCs rather than whole blood.

The low concordance between either of the IGRAs and TST results is consistent with findings of other studies, ${ }^{22}$ and reflects the likely superior specificity of the IGRAs resulting from the use of antigens not found in either BCG or NTM. ${ }^{23}$ However, the observation that nearly $30 \%$ of children with at least one IGRApositive result had a negative TST, half of whom had no measurable induration, would suggest that the TST has inferior sensitivity for demonstrating LTBI in refugee children. Moreover, $11 \%$ of attempted TSTs could not be completed, reflecting the logistical difficulties frequently encountered in reading this test, especially in high risk populations. On the basis of our data, a strategy that uses TST as a screening tool followed by a confirmatory IGRA for positive cases, as suggested by the UK NICE (National Institute for Health and Clinical Excellence) guidelines, ${ }^{24}$ is inferior to a solely IGRA-based approach.

Our findings suggest that IGRAs are the preferred screening tool for LTBI in refugee children. Both IGRAs have similar sensitivity to detect LTBI, but the discordance of positive results is sufficiently high to warrant repeat testing by the alternative IGRA to reduce false-negative results if there is strong epidemiological or clinical suspicion of $M$ tuberculosis infection. OFTGIT performance was compromised by indeterminate results in African children, especially those with other infections, whereas the T-SPOT.TB performance was compromised by the high number of failed tests, especially in younger children. Of note, an indeterminate or failed test with one IGRA was usually associated with a valid result for the other IGRA. An optimal testing strategy would involve both tests being available for sequential testing if necessary. Alternatively, a strategy taking account of both demographic and clinical characteristics of the child should be used when selecting a single IGRA. Despite valuable insights provided by this study, concerns remain about aspects of the clinical utility of all tests assessed and highlight the need for continued research to improve reliability of LTBI diagnosis in children from high incidence countries.

Acknowledgements We would like to thank staff in the Department of Clinical Immunology \& Immunogenetics \& PathWest Laboratory Medicine, especially Neill Hodgen (BSc), Jacqueline Dey (BSc), Steve Martin (MSc), Daniel Trajanoski (BSc) and Steve Pummer (BSC), for overseeing and performing the IGRAs, and Holly Philp (RN, BSc) and Sharon Nicholls (RN, BSc, also at the School Paediatrics and Child Health, University of Western Australia) for venesection and data collection; Miranda Odam (RN), Katja Pfafferott (PhD) and Susan Herrmann (RN, MSc) for assistance with study design and IRB submission from the Centre for Clinical Immunology \& Biomedical Statistics, Royal Perth Hospital \& Murdoch University: lastly we are indebted to the clinical staff in the Migrant Health Unit especially facilitator Evelyn Craig (RN, BSc)

Funding The research described in this manuscript has been funded by 0xford Immunotech. Oxford Immunotech was not involved in the planning or implementation of the study nor the interpretation of the data.

\section{Competing interests None.}

Ethics approval This study was approved by the Royal Perth Hospital Ethics Committee (EC 2007/014).

Contributors The study was conceived by ML, JW and DB, designed by ML, DB, JW EM and MF, and overseen by MF. Clinical data were collected by PN and AT and laboratory assays conducted by RW. All authors contributed to data interpretation, with statistical analysis undertaken by EM. ML, PN, EM, AL, DB and MF made key 
contributions to early drafts of the manuscript, with all authors involved in its critical review and providing final approval.

Provenance and peer review Not commissioned; externally peer reviewed.

\section{REFERENCES}

1. Roche PW, Krause V, Konstantinos A, et al. Tuberculosis notifications in Australia, 2006. Commun Dis Intell 2008:32:1-11.

2. Haas DW. Mycobacterium tuberculosis. In: Mandell GL, Bennett JE, Dolin R, eds. Principles and practice of infectious diseases. New York: Churchill Livingstone; 2000:2576-607.

3. Mandalakas AM, Starke JR. Current concepts of childhood tuberculosis. Semin Pediatr Infect Dis 2005;16:93-104.

4. National Health Service UK. The Mantoux test: administration, reading and interpretation: London: Department of Health Publications, 2005:1-6.

5. Pai M, Zwerling A, Menzies D. Systematic review: T-cell-based assays for the diagnosis of latent tuberculosis infection: an update. Ann Intern Med 2008;149:177-84

6. Connell TG, Ritz N, Paxton GA, et al. A three-way comparison of tuberculin skin testing, QuantiFERON-TB gold and T-SPOT.TB in children. PLOS ONE 2008; 3:e2624.

7. Ferrara G, Losi M, D'Amico R, et al. Use in routine clinical practice of two commercial blood tests for diagnosis of infection with Mycobacterium tuberculosis: a prospective study. Lancet 2006:367:1328-34.

8. Tavast E, Salo E, Seppala I, et al. IGRA tests perform similarly to TST but cause no adverse reactions: pediatric experience in Finland. BMC Res Notes 2009:2:9.

9. Victorian Department of Human Services. Management, control \& prevention of tuberculosis. Guidelines for health care providers (2002-2005). In: Division RRHACS, Victoria Government, 2002.

10. Cohen J. A coefficient of agreement for nominal scales. Educ Psychol Meas 1960:20:37-46.

11. Spitzer RL, Fleiss JL. A re-analysis of the reliability of psychiatric diagnosis. $\mathrm{Br} \mathrm{J}$ Psychiatry 1974;125:341-7.
12. Kobashi Y, Sugiu T, Shimizu $H$, et al. Clinical evaluation of the T-SPOT.TB test for patients with indeterminate results on the QuantiFERON TB-2G test. Intern Med 2009:48:137-42

13. Higuchi R, Mori M, Ozawa R, et al. Whole blood interferon-gamma assay for tuberculosis in children in Japan. Pediatr Int 2009;51:97-102.

14. Richeldi L, Ewer K, Losi M, et al. T-cell-based diagnosis of neonatal multidrugresistant latent tuberculosis infection. Pediatrics 2007:119:e1-5.

15. Elias D, Britton S, Kassu A, et al. Chronic helminth infections may negatively influence immunity against tuberculosis and other diseases of public health importance. Expert Rev Anti Infect Ther 2007;5:475-84.

16. Kassu A, Mengistu G, Ayele B, et al. HIV and intestinal parasites in adult TB patients in a teaching hospital in Northwest Ethiopia. Trop Doc 2007:37:222-4.

17. Yu CL, Liu CL, Tsai CY, et al. Prostaglandin E2 suppresses phytohemagglutinininduced immune responses of normal human mononuclear cells by decreasing intracellular glutathione generation, but not due to increased DNA strand breaks or apoptosis. Agents Actions 1993:40:191-9.

18. Passwell JH, Geiger B, Levanon $\mathrm{M}$, et al. IgE induces secretion of prostaglandin E2 by human monocytes. Clin Exp Immunol 1984;58:566-72.

19. Ben-Smith A, Gorak-Stolinska P, Floyd S, et al. Differences between naive and memory $T$ cell phenotype in Malawian and UK adolescents: a role for cytomegalovirus? BMC Infect Dis 2008;8:139.

20. Messele T, Abdulkadir M, Fontanet AL, et al. Reduced naive and increased activated CD4 and CD8 cells in healthy adult Ethiopians compared with their Dutch counterparts. Clin Exp Immunol 1999;115:443-50.

21. Müller K, Odum N, Bendtzen K. 1,25-Dihydroxyvitamin D3 selectively reduces interleukin-2 levels and proliferation of human $\mathrm{T}$ cell lines in vitro. Immunol Lett 1993;35:177-82.

22. Soysal A Turel 0, Toprak D et al. Comparison of positive tuberculin skin test with an interferon-gamma-based assay in unexposed children. Jpn J Infect Dis 2008;61:192-5.

23. Brett SJ, Kingston AE, Colston MJ. Limiting dilution analysis of the human $T$ cell response to mycobacterial antigens from BCG vaccinated individuals and leprosy patients. Clin Exp Immunol 1987;68:510-20.

24. National Institute for Health and Clinical Excellence. Tuberculosis: clinical diagnosis and management of tuberculosis, and measures for its prevention and control. London: Royal College of Physicians, 2006. 